\title{
Contrato psicológico, agotamiento y cinismo del empleado: su efecto en la rotación del personal operativo en la frontera norte mexicana
}

\author{
Griselda Reyes Flores ${ }^{1}$, Aurora Irma Maynez Guaderrama ${ }^{1,2 *}$, \\ Judith Cavazos Arroyo², Jesús Andrés Hernández Gómez ${ }^{1}$. \\ ${ }^{1}$ Universidad Autónoma de Ciudad Juárez, México \\ ${ }^{2}$ Universidad Popular Autónoma del Estado de Puebla, México
}

Recibido el 17 de junio de 2017; aceptado el 3 de octubre de 2018

Disponible en Internet el: 20 de noviembre de 2018

\section{Resumen}

La rotación de personal es un fenómeno costoso en términos de productividad y desempeño. La variable que mejor la predice es la intención de rotar, pero en México son escasos los trabajos empíricos sobre ella. Esta investigación tuvo por objetivo indagar si el agotamiento emocional, el cinismo organizacional y la violación del contrato psicológico, influyen sobre la intención de rotación. Para ello, se realizó una investigación cuantitativa, empírica y transversal, en una muestra de 201 empleados operativos en la industria maquiladora autopartista para la exportación, localizada en Ciudad Juárez, Chihuahua. Como técnica estadística se utilizó la modelación estructural PLS. Los resultados indican que el agotamiento emocional y el cinismo del empleado llevan a la intención de rotar, y comparativamente, es mayor la influencia del cinismo. Sin embargo, la relación predictiva entre la violación del contrato psicológico y la intención de rotar, se rechazó.

Código JEL: M12, M54, M59.

Palabras clave: Violación del Contrato psicológico; Cinismo; Agotamiento emocional; Intención de rotación; PLSSEM.

\footnotetext{
*Autor para correspondencia

Correo electrónico auroramaynez@yahoo.com (A. I. Maynez Guaderrama)

La revisión por pares es responsabilidad de la Universidad Nacional Autónoma de México.
} 


\section{Abstract}

Turnover is a costly phenomenon in terms of productivity and performance. Their best predictor is turnover intention, but in Mexico there are few empirical works about it. The purpose of this research was to investigate whether emotional exhaustion, organizational cynicism and psychological contract violation influence the turnover intention. For this purpose, a quantitative, empirical and cross-sectional investigation was carried out in a sample of 201 operative employees in the auto-parts maquiladora industry for export, located in Ciudad Juárez, Chihuahua. PLS structural modeling was used as the preferred statistical technique. The results indicate that the emotional exhaustion and cynicism of the employee lead to turnover intention and, comparatively, cynicism's influence was greater. Nevertheless, the predictive relationship between psychological contract violation and turnover intention was rejected.

JEL code: M12, M54, M59

Keywords: Psychological Contract Violation; Cynicism; Emotional Exhaustion; Turnover Intention; PLS-SEM.

\section{Introducción}

Los recursos humanos son fundamentales para establecer y mantener ventajas competitivas (Hsu, 2008; Kadiresan, Selamat, Selladurai, Ramendran, \& Mohamed, 2015; Ordoñez de Pablos \& Lytras, 2008). Las prácticas asociadas con su gestión pueden no solo ayudar a la creación y mantenimiento del capital humano organizacional, sino también dar soporte fundamental para la estrategia competitiva (Delery \& Roumpi, 2017; Huselid, 1995). Desafortunadamente, si dichas prácticas son deficientes, se puede afectar profundamente a la empresa (Long \& Perumal, 2014).

La renuncia y cobertura de vacantes es uno de los mayores costos en las empresas; en algunos trabajos sobre la rotación del personal, se identifica su influencia negativa sobre la productividad y la moral de los empleados (Reiche, 2008; Rodríguez, López, Forero, \& Gómez, 2013). Cuando los empleados se van, se pierde talento y conocimiento, situación que afecta el desempeño organizacional y los rendimientos económicos (Campbell, Coff, \& Kryscynski, 2012; Chang, Wang, \& Huang, 2013; Khanin, 2013). Luego, tanto en términos teóricos como empíricos, la rotación de personal es un fenómeno relevante, con alta significancia económica y organizativa, con interés permanente en su estudio (Harris, Kacmar, \& Witt, 2005; Long \& Perumal, 2014).

La intención de rotación es el predictor más fuerte de la rotación de personal (Hom, Mitchell, Lee, \& Griffeth, 2012; Lambert, Cluse-Tolar, Pasupuleti, Prior, \& Allen, 2011; Mobley, Griffeth, Hand, \& Meglino, 1979; Oluwafemi, 2013; Porter \& Steers, 1973; Tett \& Meyer, 1993). Entre las variables que la explican se citan el agotamiento emocional, el cinismo del empleado y la violación del contrato psicológico: por una parte, los trabajadores que se agotan emocionalmente tienden a disminuir sus esfuerzos y contribuciones para la organización; por otra, desarrollan cinismo hacia la empresa a consecuencia de largas horas de labor, trabajo intenso, estilos de liderazgos inefectivos, y continuas reducciones de la plantilla 
laboral; finalmente, el incumplimiento del contrato psicológico provoca que tengan actitudes y comportamientos negativos. Tales situaciones pueden derivar en intenciones de abandono o renuncia (Cartwright \& Holmes, 2006; Cassar, 2001; Feldman, 2000; C. Huang, Chuang, \& Lin, 2003; Hur, IL Park, \& Moon, 2014). Sin embargo, si se identifican a tiempo, las empresas pueden diseñar intervenciones organizativas apropiadas (Chang et al., 2013).

En México, los trabajadores enfrentan condiciones laborales que producen estrés crónico (Villavicencio-Ayub, Jurado-Cárdenas, \& Valencia-Cruz, 2014), problema grave y en constante crecimiento [75\% de la población lo padece] (Maldonado, Camacho, Torres, Alcaraz, \& Limón, 2015). Este fenómeno, conjuntamente con la sobrecarga de trabajo, provocan agotamiento emocional (Maslach, Schaufeli, \& Leiter, 2001; Yang, Tsai, \& Tsai, 2014) y cinismo organizacional (Erkutlu \& Chafra, 2017), e incluso pueden llevar a la eventual violación del contrato psicológico (Thomas \& Gupta, 2018). Desgraciadamente, en el país son pocos los trabajos de investigacion realizados, y generalmente se limitan a profesionales de la salud [principalmente enfermeras y médicos], con diseños de investigacion observacionales (Juárez, Idrovo, Camacho, \& Placencia, 2014).

Por ello, en empresas mexicanas del sector autopartista exportador, el objetivo de este trabajo fue determinar si la violación del contrato psicológico, el cinismo del empleado y el agotamiento emocional influyen sobre la intención de rotación del personal que labora en posiciones operativas. El documento se organiza de la siguiente manera: se presenta un marco teórico sobre la violación del contrato psicológico, el cinismo del empleado, el agotamiento emocional y sus relaciones con la intención de rotación de empleo; seguido de ello, se presenta la metodología de investigación utilizada, el análisis de resultados, la discusión y finalmente, las conclusiones del trabajo.

\section{Marco teórico}

En el contexto mexicano, las ciudades fronterizas del norte se convirtieron en un lugar atractivo para la Industria Manufacturera, Maquiladora y de Servicios de Exportación [INMEX]; entre ellas destaca Ciudad Juárez, localidad que cambió sustancialmente desde mediados de los años sesenta, cuando se implementó el Programa de Industrialización Fronteriza (Carrillo, 2001; Carrillo \& Hinojosa, 2001; Dutrénit \& Vera-Cruz, 2009; Villavicencio \& Casalet, 2005). Al mes de abril del 2016, el 60.6\% del empleo en la ciudad se generaba por la INMEX; en ella se empleaba a 253,328 personas [230,204 personal operativo incluidos obreros y técnicos], en 324 establecimientos (Plan Estratégico de Juárez, 2016).

La rotación de personal es un reto para las empresas juarenses. En ese entorno, el exceso de demanda de empleados operativos provocó rotación de personal: las personas cambiaban fácilmente de empleo al encontrar mejores ofertas laborales (Plan Estratégico de Juárez, 2016). Ante esta situación, las compañías implementaron estrategias [como el incremento de prestaciones, la inclusión de servicios de asistencia social, la contratación de personal por adelantado y los acuerdos inter-empresas para ofrecer prestaciones similares], tratando de retener a sus empleados y disminuir sus índices de rotación (Córdova, 2015), pero las iniciativas no fueron del todo exitosas, ya que las tasas de rotación se mantuvieron elevadas (Flores, 2015). En Ciudad Juárez, los índices de rotación en la industria maquiladora fueron en ascenso: en abril del 2014 eran de 3.44\% y en marzo del 2018, de 6.68\% (Ávila, 2015, 2018). 
Por otra parte, aunque la naturaleza del trabajo operativo y la de los servicios es distinta, en ambos contextos el efecto de la carga de trabajo puede llevar a manifestaciones similares de agotamiento emocional y cinismo organizacional, que eventualmente pueden llevar a los empleados a tener la intención de rotar. Por las presiones para cumplir metas de producción [a las que frecuentemente son sometidos los operadores, en muchas ocasiones para subsanar errores no necesariamente generados por ellos], se crean condiciones proclives a la modificación de acuerdos tácitos previos, que pueden llevar a la ruptura y eventual violacion del contrato psicológico; estas condiciones también fomentan en el ánimo de los trabajadores, la búsqueda de otro empleo. Por el contexto estudiado, se considera que este trabajo puede incrementar el conocimiento sobre los temas, particularmente en puestos operativos, entorno relegado en la literatura académica.

\section{Intención de rotación}

Esta variable, también conocida como intención de salida o abandono (Berry, 2010), es la que mejor predice a la rotación de personal y por ello existe un interés cada vez mayor en su estudio (Griffeth, Hom, \& Gaertner, 2000; Hom et al., 2012; Lambert et al., 2011; Mobley et al., 1979; Oluwafemi, 2013; Porter \& Steers, 1973; Tett \& Meyer, 1993). Se define como el precursor inmediato del comportamiento pensado (Mobley et al., 1979). Este constructo refleja la probabilidad subjetiva de que, dentro de cierto periodo de tiempo, el empleado deje la organización (Zhao, Wayne, Glibkowski, \& Bravo, 2007), por lo cual se le considera una consecuencia de la voluntad deliberada de abandono (Tett \& Meyer, 1993).

Sin duda, la intención de rotación es negativa para las firmas. El fenómeno afecta a las organizaciones ya que disminuye la rentabilidad [por los costos asociados de capacitación y entrenamiento (Oluwafemi, 2013) y el reemplazo y selección de personal (Surienty, Ramayah, Lo, \& Tarmizi, 2014)], puede llevar a perder conocimiento, información y talento humano valioso (Hwang, Lee, Park, Chang, \& Kim, 2014; Khanin, 2013), disminuye la moral de los empleados que permanecen en la empresa (Surienty et al., 2014), e incide negativamente sobre el liderazgo, el trabajo en equipo, y la eficacia de la organización (Harris et al., 2005), porque los empleados con intenciones de salir exhiben diversos tipos de comportamientos de abandono (Chang et al.,2013).

\section{Contrato psicológico}

La teoría del contrato psicológico es un marco referencial útil para comprender el comportamiento de los trabajadores (Zhao et al., 2007). En trabajos recientes (Restubog, Zagenczyk, Bordia, Bordia, \& Chapman, 2015) se afirma que el manejo exitoso de la relación entre empleado y empleador, depende del grado en que la empresa cumpla con el contrato psicológico, constructo clave para entender las actitudes de la gente hacia el trabajo y la empresa, su comportamiento y bienestar psicológico (Bal, De Cooman, \& Mol, 2013; Silla, Gracia, Ferreira, \& Silla, 2006).

El contrato psicológico es distinto del legal. El primero es más subjetivo que el segundo (Zhao et al., 2007). Incluye las creencias del trabajador sobre los términos, condiciones y obligaciones de un acuerdo de intercambio entre él y la empresa (Robinson \& Rousseau, 1994; Rousseau \& Greller, 1994; Rousseau \& Tijoriwala, 1998); es una promesa implícita en la que las partes se obligan mutuamente: el empleado, se compromete a trabajar duro y 
a recibir capacitación y entrenamiento; la empresa, debe recompensar el trabajo a través de la remuneración, la promoción y las oportunidades de crecimiento (Robinson \& Rousseau, 1994). En este acuerdo, las expectativas del empleado se basan en promesas percibidas [no necesariamente reconocidas por los directivos], a nivel organizacional-no se trata de una impresión respecto a una persona, gerente o supervisor-, es decir, desde la óptica del trabajador, la empresa asume una identidad antropomórfica (Morrison \& Robinson, 1997).

La violación del contrato psicológico puede provocar que el trabajador tenga la intención de rotar. Los empleados tienen reacciones emocionales asociadas al desarrollo cotidiano de tareas: cuando perciben que la empresa falló o incumplió con lo prometido implícitamente, pueden darse rupturas que eventualmente pueden llevarlos a percibir que el contrato psicológico se violó (Robinson \& Rousseau, 1994; Zhao et al., 2007). Desafortunadamente, tanto la ruptura como la violación del contrato psicológico tienen consecuencias negativas, las cuales pueden incrementar la tendencia de abandonar la empresa: disminuyen la confianza del empleado hacia la firma, incrementan el estrés, causan temores [como el no subir de puesto o perder el empleo], y provocan sentimientos de insatisfacción, defraudación y traición (Kraak, Lunardo, Herrbach, \& Durrieu, 2017; Latorre, Guest, Ramos, \& Gracia, 2016; Robinson \& Morrison, 2000; Robinson \& Rousseau, 1994; Rousseau, 1989; Stoner \& Gallagher, 2010; Zhao et al., 2007). Por lo anterior, se propone que:

H1. La violación del contrato psicológico influye positiva y significativamente sobre la intención de rotación.

\section{Cinismo del empleado}

Los nuevos desafíos competitivos han incrementado la complejidad en los entornos laborales. La estructura del trabajo ha venido cambiando: por una parte, los puestos son más ambiguos e incluyen tareas que pueden variar significativamente en periodos cortos de tiempo, con un rango cada vez más amplio; por la otra, las relaciones laborales son de corto plazo, con mayores exigencias de desempeño, sin que ello genere mejoras en salarios, puestos o compensaciones (Cardy, Gove, \& DeMatteo, 2000; Pino, del Campo, \& Ibarra, 2012). Particularmente en el personal operativo, existen fuertes presiones para laborar en ambientes donde se exige el cumplimiento de estándares elevados de calidad, se demandan altos grados de concentración, y se deja de lado la necesidad de enriquecer los puestos laborales. Por los altos niveles de tensión psicológica, los empleados se sienten frustrados y desilusionados, piensan que los recursos que se les proveen son insuficientes para atender sus tareas, comienzan a cuestionar la eficiencia e imparcialidad de los procedimientos organizacionales, e incluso desconfían y desarrollan sentimientos negativos, tanto hacia sus compañeros de trabajo como hacia la empresa (Andersson \& Bateman, 1997; Chiaburu, Peng, Oh, Banks, \& Lomeli, 2013; Thomas \& Gupta, 2018).

El cinismo del empleado es un aspecto desfavorable para las firmas. Sin importar su clasificación [organizacional, del empleado, social u organizacional] (Çınar, Karcioğlu, \& Aslan, 2014), es un fenómeno altamente peligroso (Kang, Twigg, \& Hertzman, 2010), ya que la actitud negativa asociada al mismo puede aquejar a los empleados y en la organización (Nicholson, Leiter, \& Laschinger, 2014; Salessi \& Omar, 2014). El cinismo es una respuesta 
defensiva del empleado (Cole, Bruch, \& Vogel, 2006; Pino et al., 2012), pero sus consecuencias provocan bajo desempeño laboral y comportamientos de abandono, es la dimensión opuesta del engagement (Cartwright \& Holmes, 2006; Maslach \& Leiter, 2008). A un empleado con cinismo organizacional, no le interesa su trabajo (Maslach \& Leiter, 2008). Desgraciadamente, es común que ocurra y se afirma que un número importante de trabajadores son muestran cinismo hacia la organización que los emplea (Kanter \& Mirvis, 1991; Kuo, Chang, Quinton, Lu, \& Lee, 2015).

El cinismo se puede observar en términos de la intención de rotación de los empleados; los trabajadores cínicos piensan que sus jefes no se interesan por ellos y solamente los utilizan para alcanzar los fines lucrativos de la firma (Khan, 2014). En distintas industrias y contextos, el cinismo se asocia con la intención de abandono (Çınar et al., 2014; C. Huang et al., 2003; Kang et al., 2010; Mantler, Godin, Cameron, \& Horsburgh, 2015; Wong \& Spence Laschinger, 2015), y por ello se propone que:

H2. El cinismo del empleado influye positiva y significativamente sobre la intención de rotación.

\section{Agotamiento emocional}

El agotamiento emocional es uno de los retos vigentes de la gestión organizacional (Elçi, Şener, Aksoy, \& Alpkan, 2012). Históricamente, su investigación surgió del modelo de burnout propuesto por Maslach (Cropanzano, Rupp, \& Byrne, 2003) y en trabajos recientes (Lloyd, Boer, Keller, \& Voelpel, 2015) se afirma que es uno de los determinantes relevantes del funcionamiento de la organización. El agotamiento es la fatiga resultante de la exposición a largo plazo al estrés laboral, es una manifestación crítica del mismo (Herda \& Lavelle, 2012; Yang et al., 2014).

Esta variable tiene consecuencias perjudiciales para las empresas (Cropanzano et al., 2003), ya que si se descuida el estrés de los empleados de primera línea, se pueden provocar incrementos en sus niveles de agotamiento (P.-F. Huang \& Dai, 2010), con afectaciones en la productividad, disrupción en los procesos de trabajo y disminución en la calidad de los bienes o servicios (Ghapanchi \& Aurum, 2011; P.-F. Huang \& Dai, 2010). Por ello, existe un especial interés académico y profesional por su estudio, especialmente cuando es el resultado de altas cargas laborales, presiones de tiempo y falta de apoyo social (Houkes, Janssen, de Jonge, \& Bakker, 2003).

El agotamiento emocional es un antecedente de la intención de abandonar la empresa (Hwang et al., 2014; Lambert et al., 2010; McKnight, Phillips, \& Hardgrave, 2009; ShihTse Wang, 2014). Cuando los trabajadores se sienten agotados emocionalmente, es mayor la probabilidad de que comiencen a buscar otro empleo (Cropanzano et al., 2003; Haque \& Aslam, 2014). Así, se postula que:

H3. El agotamiento emocional influye positiva y significativamente sobre la intención de rotación. 


\section{Metodología}

El diseño de la investigación fue no experimental, cuantitativo, de naturaleza empírica, transversal, con un muestreo no probabilístico por conveniencia, de acuerdo a la accesibilidad permitida. Este método de investigación es útil cuando se requiere un contacto directo con el sujeto de investigación y aunque desestima la representatividad de los casos, si toma en cuenta el tamaño de muestra (Hernández Sampieri, Fernández Collado, \& Baptista Lucio, 2010).

Durante los meses de noviembre y diciembre del 2015, se encuestó a trabajadores en puestos de operador del sector autopartista en industrias maquiladoras de exportación, localizadas en Ciudad Juárez, Chihuahua. Generalmente, los participantes se contactaron al concluir su turno de trabajo [83\%], en las unidades de transporte que los llevan a las cercanías de sus viviendas. Se diseñó un instrumento exprofeso, el cual se auto administró a los participantes. En él se incluyeron ítems con un formato tipo Likert, de cinco puntos de asignación de respuesta, dónde 1 significó "totalmente en desacuerdo" y 5 "totalmente de acuerdo". En total se recabaron 201 cuestionarios. Este tamaño de muestra cumple el criterio recomendado por Hair Jr., Hult, Ringle, and Sarstedt (2016), quienes sugieren un mínimo de 75 observaciones para detectar una $\mathrm{r}^{2}$ de 0.25 con un nivel de significancia del $1 \%$ y una potencia estadística del $80 \%$, en el caso del modelado de Ecuaciones Estructurales con Cuadrados Mínimos Parciales [PLS-SEM].

En el cuestionario se adaptaron escalas disponibles en la literatura académica. La intención de rotación se midió con 6 ítems de la escala de Ganesan y Weitz (1996). El agotamiento emocional, con 8 ítems de la escala de Maslach y Jackson (1981). El cinismo con 6 ítems de la escala de Moreno-Jiménez, Rodríguez-Carvajal y Escobar-Redonda (2001). Finalmente, la violación del contrato psicológico, se valoró con 4 ítems de la escala de Robinson y Morrison (2000). Del modelo originalmente propuesto, solamente se eliminó el ítem [Introt1], por mostrar una carga factorial menor a la recomendada. Los ítems de cada una de las variables, se presentan en la tabla 1.

Como técnica de análisis estadístico, se utilizó la modelación de ecuaciones estructurales [SEM] con mínimos cuadrados parcializados [PLS], la cual es adecuada para probar hipótesis de tipo predictor entre variables y se considera útil para analizar datos y tratar muestras pequeñas que no se ajustan a criterios paramétricos (Lowry \& Gaskin, 2014). Se aplicó el análisis de dos etapas sugerido por Anderson and Gerbing (1982). En una primera etapa, para asegurar la confiabilidad y validez de constructo, se revisó el modelo de medición [externo]; posteriormente, se analizó el modelo estructural [interno] para evaluar la relevancia predictiva del modelo y con ello probar las hipótesis de investigación (Hair Jr. et al., 2016). El software estadístico utilizado fue Smart PLS 3. 
Tabla 1

Operacionalización de las variables latentes.

\begin{tabular}{|c|c|c|c|}
\hline Intención de rotación & Agotamiento emocional & Cinismo del empleado & $\begin{array}{l}\text { Violación del contrato } \\
\text { psicológico }\end{array}$ \\
\hline $\begin{array}{l}\text { Creo que no subiré de } \\
\text { puesto en esta empresa } \\
\text { [Introt1] } \\
\text { Tengo la intención de } \\
\text { dejar esta empresa en } \\
\text { poco tiempo [Introt2]. } \\
\text { He decidido dejar esta } \\
\text { empresa [Introt3]. } \\
\text { Estoy actualmente } \\
\text { buscando trabajo como } \\
\text { el que hago, pero en otra } \\
\text { empresa [Introt4]. } \\
\text { Buscaré otro trabajo, en } \\
\text { caso de no subir de puesto } \\
\text { [Introt5]. } \\
\text { Me encuentro buscando } \\
\text { trabajo en la misma área } \\
\text { [Introt6]. }\end{array}$ & $\begin{array}{l}\text { Me siento } \\
\text { emocionalmente agotado } \\
\text { por mi trabajo [Agot1]. } \\
\text { Me siento cansado al final } \\
\text { de la jornada de trabajo } \\
\text { [Agot2]. } \\
\text { Me siento fatigado } \\
\text { cuando me levanto por la } \\
\text { mañana y tengo que ir a } \\
\text { trabajar [Agot3]. } \\
\text { Trabajar todo el día, es } \\
\text { realmente estresante para } \\
\text { mí [Agot4]. } \\
\text { Me siento cansado por mi } \\
\text { trabajo [Agot5]. } \\
\text { Me siento frustrado por } \\
\text { mi trabajo [Agot6]. } \\
\text { Creo que estoy trabajando } \\
\text { demasiado [Agot7]. } \\
\text { Trabajar directamente } \\
\text { con personas me produce } \\
\text { estrés [Agot8]. }\end{array}$ & $\begin{array}{l}\text { Desde que comencé este } \\
\text { trabajo, he ido perdiendo } \\
\text { mi interés en él [Cinis1]. } \\
\text { He ido perdiendo el } \\
\text { entusiasmo en mi trabajo } \\
\text { [Cinis2]. } \\
\text { Sólo quiero hacer mi } \\
\text { trabajo y que no me } \\
\text { molesten [Cinis3]. } \\
\text { Me burlo de mí mismo } \\
\text { pensando que mi trabajo } \\
\text { "sirve para algo" [Cinis4]. } \\
\text { Dudo sobre el valor de lo } \\
\text { que realizo en mi trabajo } \\
\text { [Cinis5]. } \\
\text { Creo que mi trabajo no } \\
\text { sirve para nada [Cinis6]. }\end{array}$ & $\begin{array}{l}\text { Respecto de la empresa en la } \\
\text { que trabajo: } \\
\text { Siento mucho coraje hacia } \\
\text { ella [Contr1]. } \\
\text { Me siento traicionado por } \\
\text { ella [Contr2]. } \\
\text { Siento que no ha cumplido } \\
\text { con los ofrecimientos que } \\
\text { me hizo [Contr3]. } \\
\text { Me siento muy frustrado por } \\
\text { cómo he sido tratado en ella } \\
\text { [Contr4]. }\end{array}$ \\
\hline
\end{tabular}

Fuente: elaboración propia

Respecto del modelo de medición, se evaluaron las cargas factoriales, los coeficientes alfa de Cronbach, los índices de fiabilidad compuesta y los indicadores de varianza extraída [AVE]. En el primer caso, las cargas factoriales mostraron valores entre 0.618 y 0.897 , con valores $t$ mayores a 1.96, lo que confirma su significancia estadística a un nivel de confianza del $95 \%$. Por otra parte, como se muestra en la tabla 2, los indicadores alfa de Cronbach se ubicaron entre 0.819 y 0.893 , con indicadores de fiabilidad compuesta [IFC] de entre 0.873 y 0.915 ; con ello, superaron los puntos de corte recomendados de 0.70 y 0.60 respectivamente (Bagozzi \& Yi, 1988). Finalmente, en todos los casos, los indicadores AVE excedieron el valor mínimo de 0.50 (Bagozzi \& Yi, 1988; Fornell \& Larcker, 1981; Seidel \& Back, 2009).

Tabla 2

Indicadores de validez convergente del modelo de medición.

\begin{tabular}{llll}
\hline Variable latente & Alfa de Cronbach & IFC & AVE \\
\hline Agotamiento & 0.893 & 0.915 & 0.576 \\
Cinismo del empleado & 0.841 & 0.882 & 0.557 \\
Intención de rotación & 0.819 & 0.873 & 0.582 \\
Violación del contrato psicológico & 0.855 & 0.903 & 0.700 \\
\hline
\end{tabular}

Fuente: elaboración propia. 
La validez discriminante se valoró en base a dos criterios: Fornell-Larcker y HeterotraitMonotrait Ratio [HTMT]. Como se muestra en la tabla 3, de acuerdo al criterio FornellLarcker, el modelo tuvo validez discriminante, porque los valores de la raíz cuadrada de AVE de las variables incluidas en el modelo [sobre la diagonal], fueron mayores que los coeficientes de correlación entre ellas (Fornell \& Larcker, 1981; Seidel \& Back, 2009). Por su parte, el indicador Heterotrait-Monotrait Ratio [HTMT] reiteró la existencia de este tipo de validez, ya que el promedio de las correlaciones entre indicadores que miden constructos distintos [heterotrait-monotrait ratios, HTMT] (Henseler, Ringle, \& Sarstedt, 2014) fueron menores del punto de corte establecido [0.85].

Tabla 3

Validez discriminante

\begin{tabular}{|l|l|l|l|l|l|l|l|}
\hline & \multicolumn{3}{|l|}{ Criterio Fornell Larcker [AVE]. } & \multicolumn{3}{l|}{ Heterotrait-Monotrait } \\
[HTMT]
\end{tabular}

Fuente: elaboración propia en base a resultados de Smart PLS 3.

\section{Resultados}

A continuación, se presentan algunos datos estadísticos descriptivos de los sujetos participantes. En su mayoría, los participantes fueron hombres [52.7\%], con una escolaridad entre primaria y secundaria [65.7\%], menores de 40 años [81.6\%], casados o en unión libre [51.8\%], adscritos al departamento de producción [96.5\%], en el primer turno [82.5\%], y con una antigüedad menor de 4 años en la empresa [81.1\%]. 
Tabla 4

Características demográficas de los sujetos de estudio

\begin{tabular}{|c|c|c|c|}
\hline Característica & & No. de Personas & Porcentaje \\
\hline \multirow[t]{3}{*}{ Sexo } & Hombre & 106 & 52.7 \\
\hline & Mujer & 95 & 47.3 \\
\hline & Total & 201 & 100.0 \\
\hline \multirow[t]{6}{*}{ Edad } & Menor de 20 & 26 & 12.9 \\
\hline & Entre 20 y 30 & 93 & 46.3 \\
\hline & Entre 31 y 40 & 45 & 22.4 \\
\hline & Entre 41 y 50 & 33 & 16.4 \\
\hline & Más de 50 & 4 & 2.0 \\
\hline & Total & 201 & 100.0 \\
\hline \multirow[t]{7}{*}{ Escolaridad } & Primaria terminada & 41 & 20.4 \\
\hline & Secundaria no terminada & 33 & 16.4 \\
\hline & Secundaria terminada & 58 & 28.9 \\
\hline & Preparatoria no terminada & 25 & 12.4 \\
\hline & Preparatoria terminada & 31 & 15.4 \\
\hline & Otra & 13 & 6.5 \\
\hline & Total & 201 & 100.0 \\
\hline \multirow[t]{5}{*}{ Estado civil } & Soltero & 88 & 43.8 \\
\hline & Casado & 49 & 24.4 \\
\hline & Divorciado/separado & 9 & 4.4 \\
\hline & Unión libre & 55 & 27.4 \\
\hline & Total & 201 & 100.0 \\
\hline \multirow[t]{5}{*}{ Antigüedad } & Menor de un año & 97 & 48.3 \\
\hline & Entre 1 y 3 años & 66 & 32.8 \\
\hline & Entre 4 y 7 años & 25 & 12.4 \\
\hline & Más de 8 años & 13 & 6.5 \\
\hline & Total & 201 & 100.0 \\
\hline
\end{tabular}

Fuente: elaboración propia.

El modelo contrastado se presenta en la figura 1, en la cual se presentan los siguientes indicadores: valores de las cargas factoriales de los constructos del modelo, coeficientes path de las relaciones estructurales y valor $\mathrm{r}^{2}$ de la intención de rotación. La calidad de predicción del modelo se valoró a partir de los coeficientes path y del valor $\mathrm{r}^{2}$. 


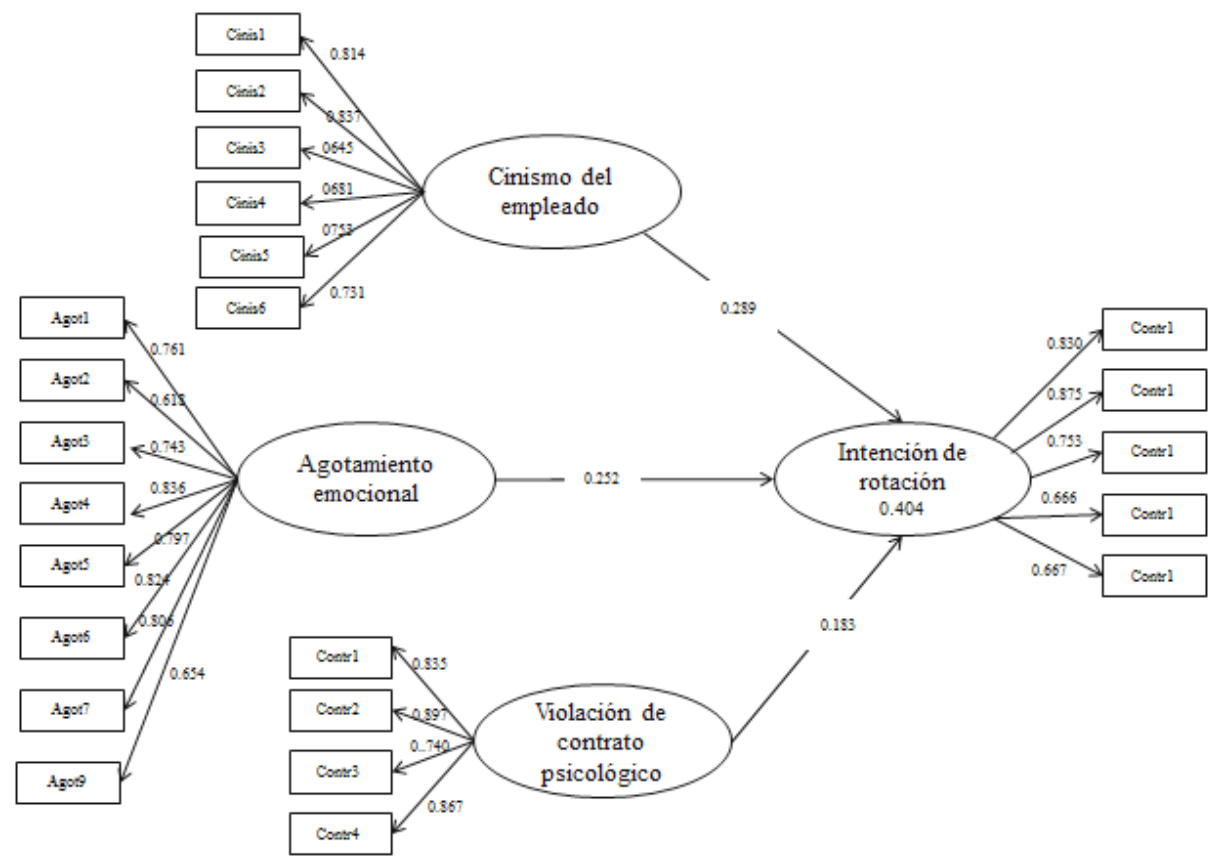

Figura 1. Modelo contrastado

Fuente: elaboración propia

Respecto de los valores path, la evaluación se realizó considerando su signo algebraico, magnitud y significancia. Cuando los indicadores path concuerdan con los postulados a priori, existe una validación empírica parcial de las relaciones teóricas propuestas (Henseler, Ringle, $\&$ Sinkovics, 2009). En este trabajo, estos valores coincidieron con los postulados previamente, pero solamente 2 de las 3 relaciones del modelo fueron estadísticamente significativas [valores t superiores a 1.96, para un nivel de confianza del $95 \%$ ] y superaron el punto de corte mínimo de 0.20 (Chin, 1998); es decir, se rechazó H1, y no se rechazaron H2 y H3 [tabla 5].

Por otra parte, el coeficiente de determinación evalúa el grado en el cual el modelo explica los datos (Seidel \& Back, 2009); así, una $\mathrm{r}^{2}$ de 0.67 se considera sustancial, una $\mathrm{r}^{2}$ de 0.35 moderada, y una $\mathrm{r}^{2}$ de 0.19 débil (Chin, 1998; Henseler et al., 2009). En ese sentido, los resultados evidenciaron que, de forma moderada-sustancial, el cinismo, el agotamiento emocional y la violación del contrato psicológico predicen a la intención de rotación $\left[\mathrm{r}^{2}=0.404\right]$, aunque como se comentó con antelación, esta última relación no fue estadísticamente significativa. 
Tabla 5

Relaciones estructurales planteadas en el modelo.

\begin{tabular}{llll}
\hline Hipótesis & Path & Valor t & Resultado \\
$\begin{array}{l}\text { H1.La violación del contrato psicológico influye positiva y significativamente } \\
\text { sobre la intención de rotación. }\end{array}$ & 0.183 & 1.848 & Rechazo \\
$\begin{array}{l}\text { H2. El cinismo del empleado influye positiva y significativamente sobre la } \\
\text { intención de rotación. }\end{array}$ & 0.289 & 2.574 & No rechazo \\
$\begin{array}{l}\text { H3: El agotamiento emocional influye positiva y significativamente sobre la } \\
\text { intención de rotación. }\end{array}$ & 0.252 & 2.938 & No rechazo \\
\hline
\end{tabular}

Fuente: elaboración propia en base a resultados de Smart PLS.

\section{Discusión y conclusiones}

En el contexto del sector autopartista de la industria maquiladora de exportación localizada en Ciudad Juárez, Chihuahua, el objetivo de este trabajo de investigación fue determinar si la violación del contrato psicológico, el agotamiento emocional y el cinismo del empleado influyen sobre la intención de rotación del personal que labora en posiciones operativas. Los resultados indican que las dos últimas variables influyen de forma directa y significativa.

En distintos contextos y sectores industriales, es cada vez mayor el reconocimiento de los efectos negativos del agotamiento emocional y de cinismo del empleado. Este trabajo da elementos para comprender lo anterior y los hallazgos tienen implicaciones teóricas y empíricas. En el entorno de la industria maquiladora de exportación del sector autopartista, el agotamiento emocional y el cinismo del empleado llevan a la intención de rotar. De forma comparada, es mayor la influencia del cinismo sobre dicho fenómeno, lo cual coincide con trabajos previos (Wong \& Spence Laschinger, 2015).

La intención de rotación se manifestó en la decisión manifiesta de los empleados de dejar la empresa, en la intención de abandonarla, y en el momento de la investigación, de buscar empleos similares, pero en otras firmas. Por su parte, el cinismo organizacional se reflejó en la pérdida de entusiasmo e interés de los trabajadores por su trabajo, en las dudas sobre el valor del mismo; es decir, a medida que los operadores pierden interés y entusiasmo por su empleo y consideran que no existe valor en lo que hacen, es mayor su intención de rotar. Estos resultados coinciden con trabajos previos (Çınar et al., 2014; C. Huang et al., 2003; Kang et al., 2010; Mantler et al., 2015; Wong \& Spence Laschinger, 2015). Éste es un fenómeno preocupante; se asocia con un retiro psicológico del empleado que provoca falta de voluntad para el desarrollo de tareas, con afectaciones para la organización (Mantler et al., 2015). Entre las alternativas para gestionarlo se encuentran el despliegue de estilos de liderazgo que reduzcan las consecuencias del cinismo organizacional: compartir información y hacer ver a los empleados el valor que tiene su trabajo, son acciones vitales en ese sentido (Thomas \& Gupta, 2018). Atender este fenómeno, es un reto para la gestión del talento humano.

Respecto del agotamiento emocional, los empleados señalaron que trabajar todo el día era realmente estresante, se sentían frustrados por su trabajo y creían que estaban trabajando demasiado. Lo anterior coincide con trabajos previos según los cuales, en el contexto mexicano, el estrés y la sobrecarga de trabajo se encuentran entre las principales razones de la rotación del personal (Vargas, 2011); en este estudio, el agotamiento emocional fue un predictor significativo de la intención de abandonar la empresa (Cropanzano et al., 2003; Haque \& Aslam, 2014; Hwang 
et al., 2014; Lambert et al., 2010; McKnight et al., 2009; Shih-Tse Wang, 2014). Por ello, se recomienda que se realicen intervenciones organizacionales, en cuanto se detecten los primeros signos de agotamiento en el empleado (Kang et al., 2010). Una alternativa es el desarrollo de líderes éticos, que escuchen a los empleados. Al respecto, trabajos muy recientes (Dust, Resick, Margolis, Mawritz, \& Greenbaum, 2018) indican que este tipo de líderes ayudan a los subordinados a darle sentido y comprensión del impacto de su trabajo, los aprecian y respetan y con ello les ayudan a sentirse parte integral de la empresa, a percibirse no como medios de producción, sino como activos valiosos para la firma. De forma paralela, se recomienda que los supervisores desarrollen su capacidad de escuchar a los subordinados, ya que ello también ayuda a enfrentar al agotamiento (Lloyd et al., 2015). Además, en el entorno puede resultar útil implementar esquemas de reconocimiento laboral, y explorar alternativas de autonomía y empoderamiento. Ello, ya que los trabajadores que concluyen su trabajo satisfactoriamente son más proclives a tener un sentimiento de logro y si se les permite explorar nuevas formas de trabajar, se verá incrementada su curiosidad, conocimiento y habilidades; todo lo anterior, incrementará sus niveles de energía (Spreitzer, Sutcliffe, Dutton, Sonenshein, \& Grant, 2005).

A diferencia de lo esperado, en esta investigación la violación del contrato psicológico no influyó sobre la intención de rotación. Aunque es recomendable continuar indagando sobre la relación, los resultados indican que el quebrantamiento del acuerdo de los términos, condiciones y obligaciones entre la empresa y el trabajador, no impacta a la intención de rotar. Una posible explicación se encuentra en la poca antigüedad de los trabajadores, situación que pudiera evitar que se llegue hasta la violación del contrato. Lo anterior, porque el tiempo es un factor relevante en los contratos psicológicos (Bal, Hofmans, \& Polat, 2016); éstos se ajustan con el paso del tiempo, conforme el empleado vive distintas experiencias en la empresa (Robinson \& Rousseau, 1994; Rousseau \& McLean Parks, 1993). Además, los hallazgos pudieran estar vinculados con los sujetos estudiados, ya que generalmente sus expectativas están acotadas al salario y prestaciones acordadas, condiciones laborales que difícilmente se incumplirán (Cortés Castillo, 2015); es decir, se trata de contratos transaccionales (Rousseau \& McLean Parks, 1993). Aun cuando la relación entre la violación del contrato psicológico y la intención de rotación no fue significativa, resulta pertinente que las empresas pongan especial cuidado durante el reclutamiento y selección de su personal [ahí comienza a formarse el contrato psicológico], y tengan siempre presentes las expectativas y promesas hechas al trabajador, tanto durante su contratación como en su vida laboral, a efecto de evitar la ruptura y posterior violación del contrato psicológico. Solamente deben hacerse promesas realistas que puedan cumplirse (Kraak et al., 2017).

A semejanza de otros, este trabajo de investigación tiene algunas limitaciones. Se trata de un estudio de corte transversal, con una muestra no probabilística, realizado en el sector autopartista de la industria manufacturera y maquiladora de exportación ubicada en Ciudad Juárez, Chihuahua, por los que los resultados no pueden ser generalizados y solo aplican en la muestra estudiada. Segundo, el análisis no considera el efecto moderador de variables demográficas, como el sexo o el estado civil, las cuales pudieran generar diferencias entre grupos, por lo que es imposible descartar la heterogeneidad de la información. Por otra parte, en relación a la muestra, se sugiere replicar el trabajo de forma probabilística, ya que, aunque el tamaño de la muestra utilizado fue estadísticamente apropiado para los propósitos del estudio, solo representa una pequeña proporción del personal ocupado en el contexto. Finalmente, para incrementar la validez externa de los resultados se recomienda llevar a cabo estudios 
longitudinales, en otros contex tos y sectores, y continuar indagando en las relaciones planteadas, particularmente en empleos distintos a los servicios humanos y de atención a la salud.

\section{Referencias}

Anderson, J. C., \& Gerbing, D. W. (1982). Some methods for respecifying measurement models to obtain unidimensional construct measurement. Journal of Marketing Research, 19(4), 453-460. doi: https://doi. org/10.1177/002224378201900407

Andersson, L. M., \& Bateman, T. S. (1997). Cynicism in the Workplace: Some Causes and Effects. Journal of Organizational Behavior, 18(5), 449-469. doi: https://doi.org/10.1002/(sici)1099-1379(199709)18:5\%3C449::aid-job808\%3E3.0.co;2-o

Ávila, C. (2015). Auge en empleo 'dispara' rotación. El Diario. Retrieved Enero, 17, 2019, from http://diario.mx/ Economia/2015-05-28_e229fc48/auge-en-empleo-dispara-rotacion/

Ávila, C. (2018). ... y se dispara rotación en maquilas arneseras. El Diario. Retrieved Enero 17, 2019, from http:// diario.mx/Economia/2018-06-17_9e316f25/-y-se-dispara-rotacion-en-maquilas-arneseras/

Bagozzi, R. P., \& Yi, Y. (1988). On the Evaluation of Structural Equation Models. Journal of the Academy of Marketing Science, 16 (1), 74-94. doi: https://doi.org/10.1007/bf02723327

Bal, P. M., De Cooman, R., \& Mol, S. T. (2013). Dynamics of psychological contracts with work engagement and turnover intention: The influence of organizational tenure. European Journal of Work \& Organizational Psychology, 22(1), 107-122. doi: https://doi.org/10.1080/1359432x.2011.626198

Bal, P. M., Hofmans, J., \& Polat, T. (2016). Breaking Psychological Contracts with the Burden of Workload: A Weekly Study of Job Resources as Moderators. Applied Psychology, 66(1), 143-167. doi: https://doi.org/10.1111/ apps.12079

Berry, M. L. (2010). Predicting Turnover Intent: Examining the Effects of Employee Engagement, Compensation Fairness, Job Satisfaction, and Age. Retrieved Enero 17, 2019, from https://trace.tennessee.edu/cgi/viewcontent. cgi?referer=https://www.google.com/\&httpsredir=1\&article=1138\&context=utk_graddiss

Campbell, B. A., Coff, R., \& Kryscynski, D. (2012). Rethinking sustained competitive advantage from human capital. The Academy of Management Review, 37(3), 376-395. doi: https://doi.org/10.5465/amr.2010.0276

Cardy, R. L., Gove, S., \& DeMatteo, J. (2000). Dynamic and customer-oriented workplaces: implications for HRM practice and research. Journal of Quality Management, 5(2), 159-186. doi: https://doi.org/10.1016/s1084$8568(01) 00019-0$

Carrillo, J. (2001). Maquiladoras de exportación y la formación de empresas mexicanas exitosas. In E. Dussel Peters (Ed.), Claroscuros. Integración exitosa de las pequeñas y medianas empresas en México (Primera ed., pp. 157208). México, D.F.: Editorial Jus, S.A. de C.V.

Carrillo, J., \& Hinojosa, R. (2001). Cableando el norte de México: la evolución de la industria maquiladora de arneses. Región y sociedad, 13(21), 79-116. doi: https://doi.org/10.22198/rys.2001.21.a734

Cartwright, S., \& Holmes, N. (2006). The meaning of work: The challenge of regaining employee engagement and reducing cynicism. Human Resource Management Review, 16(2), 199-208. doi: https://doi.org/10.1016/j. hrmr.2006.03.012

Cassar, V. (2001). Violating psychological contract terms amongst Maltese public service employees: occurrence and relationships. Journal of Managerial Psychology, 16(3), 194-208. doi: https://doi.org/10.1108/02683940110385749

Chang, W. J., Wang, Y.-S., \& Huang, T.-C. (2013). Work Design-Related Antecedents of Turnover Intention: A Multilevel Approach. Human Resource Management, 52(1), 1-26. doi: https://doi.org/10.1002/hrm.21515

Chiaburu, D. S., Peng, A. C., Oh, I.-S., Banks, G. C., \& Lomeli, L. C. (2013). Antecedents and consequences of employee organizational cynicism: A meta-analysis. Journal of Vocational Behavior, 83, 181-197. doi: https://doi. org/10.1016/j.jvb.2013.03.007

Chin, W. W. (1998). The Partial Least Squares Approach of Structural Equation Modeling In G. A. Marcoulides (Ed.), Modern Methods for Business Research (pp. 295-233). Mahwah, New York: Psychology Press. 
Çınar, O., Karcıoğlu, F., \& Aslan, İ. (2014). The Relationships among Organizational Cynicism, Job Insecurity and Turnover Intention: A Survey Study in Erzurum/Turkey. Procedia - Social and Behavioral Sciences, 150(15), 429437. doi: https://doi.org/10.1016/j.sbspro.2014.09.045

Cole, M., S., Bruch, H., \& Vogel, B. (2006). Emotion as Mediators of the Relations between Perceived Supervisor Support and Psychological Hardiness on Employee Cynicism. Journal of Organizational Behavior, 27(4), 463. doi: https://doi.org/10.1002/job.381

Córdova, C. (2015). Maquilas de Ciudad Juárez ofertan 8 mil vacantes. Retrieved Noviembre 10, 2015, from http:// www.elfinanciero.com.mx/economia/maquilas-de-ciudad-juarez-ofertan-mil-vacantes.html

Cortés Castillo, Y. S. (2015). Mujeres trabajadoras de maquiladoras en Ciudad Juárez, Chihuahua y Ciudad Victoria, Tamaulipas, México. Un análisis comparativo de sus condiciones laborales. Sinapsis-Revista de Investigación de la Escuela de Administración y Mercadotecnia del Quindio EAM, 7(7), 218-232.

Cropanzano, R., Rupp, D. E., \& Byrne, Z. S. (2003). The relationship of emotional exhaustion to work attitudes, job performance, and organizational citizenship behaviors. Journal of Applied Psychology, 88(1), 160-169. doi: https:// doi.org/10.1037/0021-9010.88.1.160

Delery, J. E., \& Roumpi, D. (2017). Strategic human resource management, human capital and competitive advantage: is the field going in circles? Human Resource Management Journal, 27(1), 1-21. doi: https://doi.org/10.1111/17488583.12137

Dust, S. B., Resick, C. J., Margolis, J. A., Mawritz, M. B., \& Greenbaum, R. L. (2018). Ethical leadership and employee success: Examining the roles of psychological empowerment and emotional exhaustion. The Leadership Quarterly, 29(5), 570-583. doi: https://doi.org/10.1016/j.leaqua.2018.02.002

Dutrénit, G., \& Vera-Cruz, A. O. (2009). Derramas de conocimiento hacia instituciones. el caso de Ciudad Juárez. In G. Dutrénit (Ed.), Sistemas Regionales de Innovación: Un espacio para el desarrollo de las Pymes (Primera ed.). México, D.F.: Universidad Autónoma Metropolitana.

Elçi, M., Şener, İ., Aksoy, S., \& Alpkan, L. (2012). The Impact of Ethical Leadership and Leadership Effectiveness on Employees' Turnover Intention: The Mediating Role of Work Related Stress. Paper presented at the 8th International Strategic Management Conference. , Barcelona.

Erkutlu, H., \& Chafra, J. (2017). Leaders' narcissism and organizational cynicism in healthcare organizations. International Journal of Workplace Health Management, 10(5), 346-363. doi: https://doi.org/10.1108/ijwhm-12-2016-0090

Feldman, D. C. (2000). The Dilbert Syndrome: How Employee Cynicism about Ineffective Management is Changing the Nature of Careers in Organizations. American Behavioral Scientist, 43(8), 1286-1300. doi: https://doi. org $/ 10.1177 / 00027640021955865$

Flores, L. (2015, 23-Sept-2015). Se intensifica pirateo de técnicos Retrieved Noviembre 10, 2015, from http://eleconomista.com.mx/estados/2015/09/23/se-intensifica-pirateo-tecnicos

Fornell, C., \& Larcker, D. F. (1981). Evaluating Structural Equation Models with Unobservable Variables and Measurement Error. Journal of Marketing Research, 18(1), 39-50. doi: https://doi.org/10.1177/002224378101800104

Ganesan, S., \& Weitz, B. A. (1996). The impact of staffing policies on retail buyer job attitudes and behaviors. Journal of Retailing, 72(1), 31-56. doi: https://doi.org/10.1016/S0022-4359(96)90004-4

Ghapanchi, A. H., \& Aurum, A. (2011). Antecedents to IT personnel's intentions to leave: A systematic literature review. Journal of Systems and Software, 84(2), 238-249. doi: https://doi.org/10.1016/j.jss.2010.09.022

Griffeth, R. W., Hom, P. W., \& Gaertner, S. (2000). A meta-analysis of antecedents and correlates of employee turnover: Update, moderator tests, and research implications for the next millennium. Journal of Management, 26(3), 463-488. doi: https://doi.org/10.1177/014920630002600305

Hair Jr., J. F., Hult, G. T. M., Ringle, C. M., \& Sarstedt, M. (2016). A primer on partial least squares structural equation modeling (PLS-SEM) (Second ed.): Sage Publications, Inc.

Haque, A., \& Aslam, M. S. (2014). The Influence of Emotional Exhaustion on Intention to Stay in the Organization: Mediating Role of Affective Commitment. International Journal of Management \& Innovation, 6(1), 16-30.

Harris, K. J., Kacmar, K. M., \& Witt, L. A. (2005). An examination of the curvilinear relationship between leadermember exchange and intent to turnover. Journal of Organizational Behavior, 26(4), 363-378. doi: https://doi. org/10.1002/job.314 
Henseler, J., Ringle, C., \& Sinkovics, R. (2009). The use of partial least squares path modeling in international marketing. In R. R. Sinkovics \& P. N. Ghauri (Eds.), Advances in International Marketing (Vol. 20, pp. 277-319): Emerald Group Publishing Limited.

Henseler, J., Ringle, C. M., \& Sarstedt, M. (2014). A new criterion for assessing discriminant validity in variance-based structural equation modeling. Journal of the Academy of Marketing Science, 43(1), 115-135. doi: https://doi. org/10.1007/s11747-014-0403-8

Herda, D. N., \& Lavelle, J. J. (2012). The auditor-audit firm relationship and its effect on burnout and turnover intention. Accounting Horizons, 26(4), 707-723. doi: https://doi.org/10.2308/acch-50181

Hernández Sampieri, R., Fernández Collado, C., \& Baptista Lucio, M. d. P. (2010). Metodología de la investigación (Quinta ed.): Mc Graw Hill.

Hom, P. W., Mitchell, T. R., Lee, T. W., \& Griffeth, R. W. (2012). Reviewing employee turnover: Focusing on proximal withdrawal states and an expanded criterion. Psychological Bulletin, 138(5), 831-858. doi: https://doi.org/10.1037/ a0027983

Houkes, I., Janssen, P. P. M., de Jonge, J., \& Bakker, A. B. (2003). Specific determinants of intrinsic work motivation, emotional exhaustion and turnover intention: A multisample longitudinal study. Journal of Occupational and Organizational Psychology, 76(4), 427-450. doi: https://doi.org/10.1348/096317903322591578

Hsu, I. C. (2008). Knowledge sharing practices as a facilitating factor for improving organizational performance through human capital: A preliminary test. Expert Systems with Applications, 35(3), 1316-1326. doi: https://doi.org/10.1016/j.eswa.2007.08.012

Huang, C., Chuang, C.-H. J., \& Lin, H.-C. (2003). The role of burnout in the relationship between perceptions of organizational politics and turnover intentions. Public Personnel Management, 32(4), 519-531. doi: https://doi. org/10.1177/009102600303200404

Huang, P.-F., \& Dai, C.-W. (2010). The impacts of emotional contagion and emotional labor perception on employees' service performance. International Journal of Electronic Business Management, 8(1), 68-79.

Hur, W.-M., IL Park, S., \& Moon, T.-W. (2014). The moderating roles of organizational justice on the relationship between emotional exhaustion and organizational loyalty in airline services. Journal of Services Marketing, 28(3), 195-206. doi: https://doi.org/10.1108/jsm-07-2012-0118

Huselid, M. A. (1995). The Impact of Human Resource Management Practices on Turnover, Productivity, and Corporate Financial Performance. The Academy of Management Journal, 38(3), 635-672. doi: https://doi.org/10.2307/256741

Hwang, J., Lee, J. J., Park, S., Chang, H., \& Kim, S. S. (2014). The Impact of Occupational Stress on Employee's Turnover Intention in the Luxury Hotel Segment. International Journal of Hospitality \& Tourism Administration, 15(1), 60-77. doi: https://doi.org/10.1080/15256480.2014.872898

Juárez, G. A., Idrovo, Á. J., Camacho, Á. A., \& Placencia, R. O. (2014). Síndrome de burnout en población mexicana: Una revisión sistemática. Salud mental, 37(2), 159-176. doi: https://doi.org/10.17711/sm.0185-3325.2014.020

Kadiresan, V., Selamat, M. H., Selladurai, S., Ramendran, C. S. P. R., \& Mohamed, R. K. M. H. (2015). Performance Appraisal and Training and Development of Human Resource Management Practices (HRM) on Organizational Commitment and Turnover Intention. Asian Social Science, 11(24), 162-176. doi: https://doi.org/10.5539/ass. $\mathrm{v} 11 \mathrm{n} 24 \mathrm{p} 162$

Kang, B., Twigg, N. W., \& Hertzman, J. (2010). An examination of social support and social identity factors and their relationship to certified chefs' burnout. International Journal of Hospitality Management, 29(1), 168-176. doi: https://doi.org/10.1016/j.ijhm.2009.08.004

Kanter, D. L., \& Mirvis, P. H. (1991). Cynicism: The new American malaise. Business \& Society, 91(77), 57-61.

Khan, M. A. (2014). Organizational Cynicism and Employee Turnover Intention: Evidence from Banking Sector in Pakistan. Pakistan Journal of Commerce \& Social Sciences, 8(1), 30-41.

Khanin, D. (2013). How to reduce turnover intentions in the family business: Managing centripetal and centrifugal forces. Business Horizons, 56(1), 63-73. doi: https://doi.org/10.1016/j.bushor.2012.09.005

Kraak, J. M., Lunardo, R., Herrbach, O., \& Durrieu, F. (2017). Promises to employees matter, self-identity too: Effects of psychological contract breach and older worker identity on violation and turnover intentions. Journal of Business Research, 70, 108-117. doi: https://doi.org/10.1016/j.jbusres.2016.06.015 
Kuo, C.-C., Chang, K., Quinton, S., Lu, C.-Y., \& Lee, I. (2015). Gossip in the workplace and the implications for HR management: a study of gossip and its relationship to employee cynicism. The International Journal of Human Resource Management, 26(18), 2288-2307. doi: https://doi.org/10.1080/09585192.2014.985329

Lambert, E. G., Cluse-Tolar, T., Pasupuleti, S., Prior, M., \& Allen, R. I. (2011). A Test of a Turnover Intent Model. Administration in Social Work, 36(1), 67-84. doi: https://doi.org/10.1080/03643107.2010.551494

Lambert, E. G., Hogan, N. L., Jiang, S., Elechi, O. O., Benjamin, B., Morris, A., . . Dupuy, P. (2010). The relationship among distributive and procedural justice and correctional life satisfaction, burnout, and turnover intent: An exploratory study. Journal of Criminal Justice, 38(1), 7-16. doi: https://doi.org/10.1016/j.jcrimjus.2009.11.002

Latorre, F., Guest, D., Ramos, J., \& Gracia, F. J. (2016). High commitment HR practices, the employment relationship and job performance: A test of a mediation model. European Management Journal, 34(4), 328-337. doi: https:// doi.org/10.1016/j.emj.2016.05.005

Lloyd, K. J., Boer, D., Keller, J. W., \& Voelpel, S. (2015). Is My Boss Really Listening to Me? The Impact of Perceived Supervisor Listening on Emotional Exhaustion, Turnover Intention, and Organizational Citizenship Behavior. Journal of Business Ethics, 130(3), 509-524. doi: https://doi.org/10.1007/s10551-014-2242-4

Long, C. S., \& Perumal, P. (2014). Examining the impact of human resource management practices on employee's turnover intention. International Journal of Business \& Society, 15(1), 111-126.

Lowry, P. B., \& Gaskin, J. (2014). Partial Least Squares (PLS) Structural Equation Modeling (SEM) for Building and Testing Behavioral Causal Theory: When to Choose It and How to Use It. IEEE Transactions on Professional Communication, 57(2), 123-146. doi: https://doi.org/10.1109/tpc.2014.2312452

Maldonado, M. A., Camacho, A. R., Torres, S. G. V., Alcaraz, J. L. G., \& Limón, J. (2015). Determination of Burnout Syndrome among Middle and Senior Managers in Manufacturing Industry in Ciudad Juarez. Procedia Manufacturing, 3, 6459-6466. doi: https://doi.org/10.1016/j.promfg.2015.07.927

Mantler, J., Godin, J., Cameron, S. J., \& Horsburgh, M. E. (2015). Cynicism in hospital staff nurses: the effect of intention to leave and job change over time. Journal of Nursing Management, 23(5), 577-587. doi: https://doi. org/10.1111/jonm.12183

Maslach, C., \& Jackson, S. E. (1981). The measurement of experienced burnout. Journal of Organizational Behavior, 2(2), 99-113. doi: https://doi.org/10.1002/job.4030020205

Maslach, C., \& Leiter, M. P. (2008). Early Predictors of Job Burnout and Engagement. Journal of Applied Psychology, 93(3), 498-512. doi: https://doi.org/10.1037/0021-9010.93.3.498

Maslach, C., Schaufeli, W. B., \& Leiter, M. P. (2001). Job Burnout. Annual Review of Psychology, 52(1), 397-422. doi: https://doi.org/10.1146/annurev.psych.52.1.397

McKnight, D. H., Phillips, B., \& Hardgrave, B. C. (2009). Which reduces IT turnover intention the most: Workplace characteristics or job characteristics? Information \& Management, 46(3), 167-174. doi: https://doi.org/10.1016/j. im.2009.01.002

Mobley, W. H., Griffeth, R. W., Hand, H. H., \& Meglino, B. M. (1979). Review and conceptual analysis of the employee turnover process. Psychological Bulletin, 86(3), 493-522. doi: https://doi.org/10.1037//0033-2909.86.3.493

Moreno-Jiménez, B., Rodríguez-Carvajal, R., \& Escobar Redonda, E. (2001). La evaluación del burnout profesional. Factorialización del MBI-GS. Un análisis preliminar. Ansiedad y estrés, 7(1), 69-78.

Morrison, E. W., \& Robinson, S. L. (1997). When Employees Feel Betrayed: A Model of How Psychological Contract Violation Develops. The Academy of Management Review, 22(1), 226-256. doi: https://doi.org/10.2307/259230

Nicholson, R. M., Leiter, M. P., \& Laschinger, H. K. S. (2014). Predicting cynicism as a function of trust and civility: a longitudinal analysis. Journal of Nursing Management, 22(8), 974-983. doi: https://doi.org/10.1111/jonm.12073

Oluwafemi, O. (2013). Predictors of turnover intention among employees in Nigeria's Oil industry. Organizations \& Markets in Emerging Economies, 4(2), 42-63.

Ordoñez de Pablos, P., \& Lytras, M. (2008). Competencies and human resource management: implications for organizational competitive advantage. Journal of Knowledge Management, 12(6), 48-55. doi: https://doi. org/10.1108/13673270810913612

Pino, N. D., del Campo, A. M., \& Ibarra, D. V. (2012). Manejo del cinismo organizacional: La oportunidad del líder. Revista Iberoamericana de Psicología: Ciencia y Tecnología, 5(2), 7-16. 
Plan Estratégico de Juárez. (2016). Entre crecimiento y salarios bajos. Radiografía del empleo en Juárez Así estamos Juárez. Sistema de indicadores de calidad de vida. (pp. 54). Ciudad Juárez: Plan Estratégico de Juárez.

Porter, L. W., \& Steers, R. M. (1973). Organizational, work, and personal factors in employee turnover and absenteeism. Psychological Bulletin, 80(2), 151-176. doi: https://doi.org/10.1037/h0034829

Reiche, B. S. (2008). The configuration of employee retention practices in multinational corporations' foreign subsidiaries. International Business Review, 17(6), 676-687. doi: https://doi.org/10.1016/j.ibusrev.2008.09.004

Restubog, S. L. D., Zagenczyk, T. J., Bordia, P., Bordia, S., \& Chapman, G. J. (2015). If You Wrong Us, Sha11 We Not Revenge? Moderating Roles of Self-Control and Perceived Aggressive Work Culture in Predicting Responses to Psychological Contract Breach. Journal of Management, 41(4), 1132-1154. doi: https://doi. org/10.1177/0149206312443557

Robinson, S. L., \& Morrison, E. (2000). The development of psychological contract breach and violation: a longitudinal study. Journal of Organizational Behavior, 21(5), 525-546. doi: https://doi.org/10.1002/1099-1379(200008)21:5\%3C525::AID-JOB40\%3E3.0.CO;2-T

Robinson, S. L., \& Rousseau, D. M. (1994). Violating the psychological contract: Not the exception but the norm. Journal of Organizational Behavior, 15(3), 245-259. doi: https://doi.org/10.1002/job.4030150306

Rodríguez, S. Y., López, E. S., Forero, Y. M., \& Gómez, S. A. (2013). Rotación de personal en el call center Calldinero. Desarrollo \& Gestión(9), 9-14.

Rousseau, D. M. (1989). Psychological and implied contracts in organizations. Employee Responsibilities and Rights Journal, 2(2), 121-139. doi: https://doi.org/10.1007/BF01384942

Rousseau, D. M., \& Greller, M. M. (1994). Human resource practices: administrative contract makers. Human Resource Management, 33(3), 385-401. doi: https://doi.org/10.1002/hrm.3930330308

Rousseau, D. M., \& McLean Parks, J. (1993). The contracts of individuals and organizations. Research in Organizational Behavior, 15, 1-43.

Rousseau, D. M., \& Tijoriwala, S. A. (1998). Assessing psychological contracts: issues, alternatives and measures. Journal of Organizational Behavior, 19(S1), 679-695. doi: https://doi.org/10.1002/(sici)1099-1379(1998)19:1+\%3C679::aid-job971\%3E3.0.co;2-n

Salessi, S., \& Omar, A. (2014). Cinismo Organizacional: Consecuencias sobre la salud mental de los trabajadores. Psicología y Salud, 24(2), 269-277.

Seidel, G., \& Back, A. (2009). Success factor validation for global ERP programmes. Paper presented at the 17th European Conference on Information Systems, Verona.

Shih-Tse Wang, E. (2014). The effects of relationship bonds on emotional exhaustion and turnover intentions in frontline employees. Journal of Services Marketing, 28(4), 319-330. doi: https://doi.org/10.1108/jsm-11-2012-0217

Silla, J. M. P., Gracia, F. J., Ferreira, L. F., \& Silla, I. (2006). El estado del contrato psicológico y su relación con la salud psicológica de los empleados. Psicothema, 18(2), 256-262.

Spreitzer, G., Sutcliffe, K., Dutton, J., Sonenshein, S., \& Grant, A. M. (2005). A Socially Embedded Model of Thriving at Work. Organization Science, 16(5), 537-549. doi: https://doi.org/10.1287/orsc.1050.0153

Stoner, J. S., \& Gallagher, V. C. (2010). Who Cares? The Role of Job Involvement in Psychological Contract Violation. Journal of Applied Social Psychology, 40(6), 1490-1514. doi: https://doi.org/10.1111/j.1559-1816.2010.00626.x

Surienty, L., Ramayah, T., Lo, M.-C., \& Tarmizi, A. N. (2014). Quality of Work Life and Turnover Intention: A Partial Least Square (PLS) Approach. Social Indicators Research, 119(1), 405-420. doi: https://doi.org/10.1007/s11205013-0486-5

Tett, R. P., \& Meyer, J. P. (1993). Job satisfaction, organizational commitent, turnover intention, and turnover: Paht analyses based on meta_analytic findings. Personnel Psychology, 46(2), 259-293. doi: 10.1111/j.1744-6570.1993. tb00874.x

Thomas, N., \& Gupta, S. (2018). Organizational cynicism - what every manager needs to know. Development and Learning in Organizations: An International Journal, 32(2), 16-19. doi: https://doi.org/10.1108/DLO-01-20170005

Vargas, I. (2011). Rotación laboral enemiga de la empresa. Retrieved Septiembre 11, 2018, from http://www.cnnexpansion.com/mi-carrera/2011/02/10/empleo-rotacion-laboral-renuncia-cnn 
Villavicencio-Ayub, E., Jurado-Cárdenas, S., \& Valencia-Cruz, A. (2014). Work engagement and occupational burnout: Its relation to organizational socialization and psychological resilience. Journal of Behavior, Health \& Social Issues, 6(2), 45-55. doi: https://doi.org/10.5460/jbhsi.v6.2.47026

Villavicencio, C. D. H., \& Casalet, R. M. (2005). La construcción de un" entorno" institucional de apoyo a la industria maquiladora en la frontera norte de México. Revista galega de economía: Publicación Interdisciplinar da Facultade de Ciencias Económicas e Empresariais, 14(1), 1-20.

Wong, C. A., \& Spence Laschinger, H. K. (2015). The influence of frontline manager job strain on burnout, commitment and turnover intention: A cross-sectional study. International Journal of Nursing Studies, 52(12), 1824-1833. doi: https://doi.org/10.1016/j.ijnurstu.2015.09.006

Yang, F.-H., Tsai, Y.-S., \& Tsai, K.-C. (2014). The influences of ethical climate on turnover intention: the mediating role of emotional exhaustion. International Journal of Organizational Innovation, 6(4), 72-89.

Zhao, H., Wayne, S. J., Glibkowski, B. C., \& Bravo, J. (2007). The impact of psychological contract breach on work-related outcomes: a meta-analysis. Personnel Psychology, 60(3), 647-680. doi: https://doi.org/10.1111/j.17446570.2007.00087.x 